\title{
RECURRENT OPISTHOTONUS IN CATATONIA: AN ATYPICAL PRESENTATION
}

NARAYANA MANJUNATHA, URVAKHSH MEHERWAN MEHTA, JOHN P.

\section{ABSTRACT}

Opisthotonus is known to occur in tetanus, rabies, cerebral malaria, neurosyphilis, acute cerebral injury and other medical conditions. Opisthotonus, so far, has not been reported in any major psychiatric disorder. Authors report a case of recurrent opisthotonus presenting concurrently with other catatonic signs which showed dramatic response to combination of lorazepam and electroconvulsive therapy (ECT). Clinicians should consider the possibility of catatonia in the differential diagnosis of opisthotonus since catatonia can be treated easily with benzodiazepines and ECT.

Key words: Catatonia, opisthotonus, posturing

DOI: $10.4103 / 0019-5359.58881$

\section{INTRODUCTION}

Opisthotonus is a state of extreme hyperextension of the head, neck and spine that presents as severe, prolonged spasm characterized by a strongly arched and rigid back, hyperextended neck and arms, with the heels bent back. ${ }^{[1]}$ Opisthotonus is known to occur in tetanus, rabies, cerebral malaria, neurosyphilis, meningitis, encephalitis, cerebral palsy, partial seizures, strychnine poisoning and adverse effects of medications. ${ }^{[2-6]}$

Catatonia is a neuropsychiatric condition with unique combinations of mental, motor

Department of Psychiatry, National Institute of Mental Health and Neurosciences (NIMHANS), Bangalore, India

\section{Correspondence:}

Dr. N. Manjunatha

Department of Psychiatry,

National Institute of Mental Health and Neurosciences

(NIMHANS), Bangalore - 560 029, India

E-mail: manjunatha.adc@gmail.com and behavioral signs. ${ }^{[7]}$ Catatonic signs consist of motor abnormalities in the form of posturing, gegenhalten, waxy flexibility, echolalia/ echopraxia, rigidity, stereotypies and psychological pillow. ${ }^{[8]}$ The PUBMED index search using combination of terms like "catatonia" and "opisthotonus/ catatonia and opisthotonos" [both opisthotonus and opisthotonos are accepted in the literature, but opisthotonus (rather than -tonos) is more frequently cited and better accepted] yielded 3 case reports, ${ }^{[9-11]}$ but no authors considered opisthotonus as a catatonic sign. Authors report a case of catatonia in which opisthotonus (arc de cercle) presented as a form of posturing (which itself is a catatonic sign).

\section{CASE REPORT}

A 26-year-old formally educated married man hailing from a rural background, working as a migrant construction worker with negative past, 
family and personal history, presented to our emergency unit on the $8^{\text {th }}$ day of illness (for the first time for medical attention) of abrupt onset, characterized by a polymorphic clinical picture without precipitating factors. At the onset of illness, i.e., 8 days prior to presentation, the patient abruptly became anxious and irritable for a day. Thereafter, he absconded from home and wandered aimlessly in public places, exhibiting disinhibited behavior over the next 2 days. When he was brought back home by police on the $4^{\text {th }}$ day, he was noted to be irritable and started claiming that all family members were in danger and that the police were on their trail. Soon after being brought back home, the patient became extremely agitated, screaming loudly, running out onto the streets, hitting walls and doors with closed fists and being sleepless throughout the night. This extreme psychomotor excitement continued for the next 36 hours and then calmed down.

During the next 2 days, the clinical picture changed to mutism, posturing, withdrawal, negativism and passing urine in clothes. During this period, the patient was noted to hold his hands in a folded position continuously (posture of namasthe, the traditional way of greeting each other in Indian culture) for about 30 to 45 minutes at a time. There was no history of substance abuse, consumption/ administration of any medications (including neuroleptics) in the recent past; fever; motor weakness; loss of consciousness; seizures; recent open wound; head injury; or dog bite. There were no symptoms of mania, depression, schizophrenia or suicidal attempts/ ideation.

On examination in the emergency care unit on the $8^{\text {th }}$ day of illness, the patient had catatonic signs - stupor, mutism, withdrawal, posturing and autonomic instability. The baseline BushFrancis catatonia rating scale (BFCRS ${ }^{[12]}$ score was 25. A detailed neurological and systemic examination ruled out localizing neurological signs, hepatosplenomegaly and other clinical features that could suggest the presence of primary medical/ neurological conditions such as cerebral malaria, neurosyphilis, etc. The patient was diagnosed provisionally as having brief psychotic disorder (DSM-IV TR) with prominent catatonic signs, and was initiated on treatment with intravenous lorazepam $2 \mathrm{mg}$ thrice daily, after an initial statum intravenous dose of $4 \mathrm{mg}$. While in the hospital, the patient developed facial grimacing [due to facial muscle spasm, giving an appearance of a sneering grin like that of risus sardonicus], motor stereotypies of lower jaw, verbal stereotypies, mitgehen, posturing and psychological pillow. The above catatonic features were accompanied by episodic and recurrent opisthotonus, or arc de cercle, each episode lasting for 10 to 20 minutes, with multiple such episodes occurring over the course of 24 hours with a gap of a few minutes between episodes. These episodes of opisthotonus co-occurred with fluctuating catatonic signs and features of autonomic instability (tachycardia, fluctuating blood pressure and sweating). The patient was noted to have low-grade intermittent fever (maximum of $99^{\circ} \mathrm{F}$ ), which responded to antipyretics. This clinical picture resembled earlier descriptions of malignant/ lethal catatonia. ${ }^{[13,14]}$ Authors also ruled out psychogenic opisthotonus and myoclonic seizures in this patient by history and observation in hospital.

Baseline routine biochemical parameters 
did not reveal any abnormality. Repeat laboratory parameters after occurrence of opisthotonus, such as blood glucose, creatininephosphokinase, liver and renal parameters, electrolytes, hemogram, urine microscopy and culture, revealed no abnormality. Computed tomography scan of head, as well as cerebrospinal fluid analysis (routine, acid-fast and India ink staining with Herpes Simplex Virus antibody titers), did not reveal any abnormality. Electroencephalography showed well-defined alpha rhythm with lorazepam-induced fast beta activity and no epileptiform discharges.

The patient was maintained on parenteral nutrition and supportive medical care in the intensive care unit. Dramatic improvement was observed with intravenous lorazepam $2 \mathrm{mg}$ thrice daily and one session of modified bilateral electroconvulsive therapy (ECT). Over the next 3 days, the patient recovered completely from catatonia, with BFCRS score of zero. Lorazepam was subsequently tapered off and stopped over the next 4 days. No antipsychotics were used till the patient recovered from catatonia.

Following complete recovery from catatonia on the $13^{\text {th }}$ day of illness, the patient revealed that he wandered away from home during the initial period of excitement in response to command auditory hallucinations. The patient was discharged on risperidone $4 \mathrm{mg} /$ day with a final diagnosis of brief psychotic disorder (DSMIV). The patient has not subsequently attended the follow-up.

\section{DISCUSSION}

Understanding opisthotonus as a catatonia sign has treatment implications, especially when it is associated with other catatonic signs. Catatonia can be treated easily with lorazepam and ECT, thus avoiding extensive investigations to rule out other causes of opisthotonus.

Authors consider opisthotonus in this patient as a variant of stereotypic posturing, which is a catatonic sign. Posturing is considered as one of the more distinctive phenomena in catatonia. ${ }^{[15]}$ It is described as a specific, uncomfortable and often bizarre positioning of body parts against gravity with complete akinesia maintained for hours, days and sometimes weeks. Different forms of posturing involving limbs (classic posturing), head (psychic pillow) and eyes (staring) have been described, which can broadly be classified into two types - manneristic and stereotypic. ${ }^{[16]}$ Manneristic posture is an odd stilted posture that is an exaggeration of a normal posture and not rigidly preserved, while a stereotyped posture is an abnormal and nonadaptive posture that is rigidly maintained (e.g., psychological pillow).

Authors consider catatonic signs in this patient as part of acute psychosis (DSMIV). There were no clinical or laboratory findings suggestive of a primary underlying organic cause that could explain the above clinical picture. Neuroleptic/ drug-induced extrapyramidal side effect as cause of opisthotonus was also ruled out, as the patient had not received any neuroleptics prior to the appearance of opisthotonus (as he was brought to medical attention for the first time). However, it is our contention that irrespective of whether an underlying organic cause is established, opisthotonus in this patient could still be considered as a catatonic sign since 
it appeared along with other catatonic signs following the initial period of excitement and resolved with symptomatic treatment along with the other catatonic signs. Therefore, the authors concluded that recurrent opisthotonus in this patient was a catatonic sign, i.e., a form of catatonic posturing.

The authors propose, therefore, that the neurobiological substrate of opisthotonus is similar to that of catatonic posturing, involving a deficit in 'termination' of movements, mediated by the posterior parietal cortex. ${ }^{[17]}$ Compromised GABAergic (neuron producing gamma-aminobutyric acid) inhibition in the spinal cord has also been suggested as a possible etiological factor in opisthotonic posturing. ${ }^{[2]}$ The dramatic improvement in opisthotonic posturing following intravenous lorazepam in this patient provides strong support to the above theory.

\section{CONCLUSION}

Opisthotonus may be a catatonic sign in medical emergency. Clinicians should consider the possibility of idiopathic catatonia in their differential diagnosis of opisthotonus. Opisthotonus co-occurring with other catatonic signs in the absence of other features suggesting an underlying organic cause should be treated primarily with benzodiazepines and ECT.

\section{REFERENCES}

1. Spillane J. Involuantary movements. In: Bickerstaff's neurological examination in clinical practice. 6th ed. Oxford: Blackwell Science Ltd; 1996. p. 160-75.
2. Ries CR, Scoates PJ, Puil E. Opisthotonos following propofol: A nonepileptic perspective and treatment strategy. Can J Anaesth 1994;41:414-9.

3. Wong KT, Lum LCS, Lam SK, Huang CC, Liu CC, Chang YC. Enterovirus 71 infection and neurologic complications. $\mathrm{N}$ Engl $\mathrm{J}$ Med 2000;342:356.

4. Hendrickson RG, Morocco AP, Greenberg MI. Acute dystonic reactions to "Street Xanax". N Engl J Med 2002;346:1753.

5. Idro R, Otieno G, White S, Kahindi A, Fegan $G$, Ogutu B, et al. Decorticate, decerebrate and opisthotonic posturing and seizures in Kenyan children with cerebral malaria. Malar J 2005;7:4:57.

6. Chauhan VV, Pickens AT. Opisthotonus in neurosyphilis: A case report. J Emerg Med 2008 in press.

7. Braunig $P$, Kruger S. History. In: Caroff SN, Mann SC, Francis A, Fricchione GL, editors. Catatonia - from psychopathology to neurobiology. Washington DC: American Psychiatric Publishing Inc; 2004. p. 1-14.

8. Taylor MA. Clinical examination. In: Caroff SN, Mann SC, Francis A, Fricchione GL, editors. Catatonia - from psychopathology to neurobiology. Washington DC: American Psychiatric Publishing Inc; 2004. pp. 45-64.

9. Dekleva KB, Husain MM. Sporadic encephalitis lethargica: A case treated successfully with ECT. J Neuropsychiatry Clin Neurosci 1995;7:237-9.

10. Diesing TS, Wijdicks EF. Arc de cercle and dysautonomia from anoxic injury. Mov Disord 2006;21:868-9.

11. Engquist A, Jørgensen BC, Andersen HB. Catatonia after epidural morphine. Acta Anaesthesiol Scand 1981;25:445-6.

12. Bush G, Fink M, Petrides G, Dowling F, Francis A. Catatonia I. Rating scale and standard examination. Acta Psychiatr Scand 1996;93:12936.

13. Castillo E, Rubin RT, Holsboer-Trachsler E. Clinical differentiation between lethal catatonia 
and neuroleptic malignant syndrome. Am J Psychiatry 1989;146:324-8.

14. Mann SC, Caroff SN, Fricchione GL, Campbell C, Greenstein RA. Malignant catatonia. In: Caroff SN, Mann SC, Francis A, Fricchione GL, editors. Catatonia - from psychopathology to neurobiology. Washington DC: American Psychiatric Publishing Inc; 2004. p. 105-120.

15. Pantelis $C$, Malhi GS. Pillow talk: catatonia. Acta Neuropsychiatr 2008;20:44-5.
16. Cassey P, Kelly B. Motor disorders. In: Fish's clinical psychopthology-signs and symptoms in psychiatry. 3rd ed. London: Royal College of Psychiatrists; 2007. p. 87-105.

17. Northoff G. What catatonia can tell us about "topdown modulation": A neuropsychiatric hypothesis. Behav Brain Sci 2002;25:555-604.

Source of Support: Nil. Conflict of Interest: None declared. 